\title{
Método ABP y aprendizaje de vectores en estudiantes universitarios: una revisión de la literatura científica del 2015 - 2020
}

ABP method and vector learning in university students: a review of the scientific literature from $2015-2020$

Método ABP e aprendizagem vetorial em estudantes universitários: uma revisão da literatura científica de 2015-2020

\section{ARTÍCULO ORIGINAL}

Rubén Darío Mendoza Arenas

rmendozaar@ucvvirtual.edu.pe

https://orcid.org/0000-0002-7861-7946

Universidad Cesar Vallejo

Lima - Perú

\author{
Hugo Eladio Chumpitaz Caycho \\ hchumpitazc@ucvvirtual.edu.pe \\ https://orcid.org/0000-0001-6768-381X \\ Universidad Cesar Vallejo \\ Lima - Perú
}

Marisol Paola Delgado Baltazar

https://orcid.org/0000-0002-0278-9557

marisolpaoladelgadob@gmail.com

Universidad Nacional del Callao

Lima - Perú

Recibido 05 de Febrero 2021 | Arbitrado y aceptado 19 de Abril 2021 | Publicado 28 de Julio 2021

\section{RESUMEN}

Esta investigación permitió conocer sobre el Método ABP y el aprendizaje de vectores. Se planteó como objetivo, conocer la influencia del método basado en problemas (ABP) en el aprendizaje de vectores en estudiantes universitarios, mediante una revisión sistemática entre los años 2015 al 2020. La metodología es una revisión sistemática de artículos encontrados en la base de datos Scopus, Wos, y Scielo. Se revisaron 100 artículos y finalmente, luego de un exhaustivo filtro se seleccionaron 15 estudios.

Los resultados reflejan que, el Método de Aprendizaje basado en problemas (ABP), tiene muchas interpretaciones. Según L.A. Branda en el artículo El aprendizaje basado en problemas. De herejía artificial a res popularis; nos da una reflexión sobre el aprendizaje autodirigido y su relación con el aprendizaje autorregulado.El estudio del artículo de Carlos Rodolfo Paredes titulado "Aprendizaje basado en problemas (ABP): Una estrategia de enseñanza de la educación ambiental, en estudiantes de un liceo municipal de Cañete", estudia, organiza y presenta resultados, dado que el ABP, le permite reflexionar sobre sus aciertos y errores y mejorar sus aprendizajes a medida que conoce e indaga más sobre el tema de fotosíntesis y finalmente en México; Yosajandi Pérez Campillo y José Antonio Chamizo Guerrero en su revisión sistemática titulada: El ABP y el diagrama heurístico como herramientas para desarrollar la argumentación escolar en las asignaturas de ciencias, se introduce con alumnos de química del bachillerato mexicano, se introduce una nueva herramienta denominada Diagrama Heurístico ABP que facilita adquirir la competencia argumentativa.

Palabras Clave: Revisión Sistemática, Método $\mathrm{ABP}$, Aprendizaje de vectores.

\section{ABSTRACT}

This research allowed us to learn about the ABP Method and vector learning. The objective was to know the influence of the problem-based method (PBL) in the learning of vectors in university students, through a systematic review between the years 2015 to 2020 . The methodology is a systematic review of articles found in the database Scopus, Wos, and Scielo. 100 articles were reviewed and finally, after an exhaustive filter, 15 studies were selected.

The results reflect that the Problem-Based Learning Method (PBL) has many interpretations. According to. Branda in the article Problem-based learning. From artificial heresy to res popularis; gives us a reflection on self-directed learning and its relationship with self-regulated learning.The study of the article by Carlos Rodolfo Paredes entitled " Problembased learning (PBL): A teaching strategy of environmental education, in students of a municipal high school de Cañete ", studies, organizes and presents results, since the ABP allows him to reflect on his successes and mistakes and improve his learning as he learns and investigates more about the subject of photosynthesis and finally in Mexico; Yosajandi Pérez Campillo and José Antonio Chamizo Guerrero in their systematic review entitled: The $\mathrm{ABP}$ and the heuristic diagram as tools to develop school argumentation in science subjects, it is introduced with chemistry students of the Mexican high school, a new tool called Diagram is introduced ABP heuristic that facilitates the acquisition of argumentative competence.

Key Words: Systematic Review, PBL method, Vector learning.

\section{RESUMO}

Esta pesquisa nos permitiu conhecer o Método $\mathrm{ABP}$ e o aprendizado de vetores. $\mathrm{O}$ objetivo foi conhecer a influência do método baseado em problemas (PBL) na aprendizagem de vetores em universitários, por meio de uma revisão sistemática entre os anos de 2015 a 2020 . A metodologia é uma revisão sistemática de artigos encontrados na base de dados Scopus, Wos e Scielo. Foram revisados 100 artigos e, por fim, após filtro exaustivo, foram selecionados 15 estudos.

Os resultados refletem que o Método de Aprendizagem Baseada em Problemas (PBL) tem muitas interpretações. De acordo com. Branda no artigo Aprendizagem baseada em problemas. Da heresia artificial à res popularis; nos dá uma reflexão sobre a aprendizagem autodirigida e sua relação com a aprendizagem autorregulada. $\mathrm{O}$ estudo do artigo de Carlos Rodolfo Paredes intitulado " Aprendizagem baseada em problemas (PBL): Uma estratégia de ensino de educação ambiental, em alunos de um município o colégio de Cañete ", estuda, organiza e apresenta resultados, já que o $\mathrm{ABP}$ lhe permite refletir sobre seus acertos e erros e melhorar seu aprendizado à medida que aprende e investiga mais sobre o tema da fotossíntese e finalmente no México; Yosajandi Pérez Campillo e José Antonio Chamizo Guerrero em sua revisão sistemática intitulada: $\mathrm{O} A B P$ e o diagrama heurístico como ferramentas para desenvolver a argumentação escolar em disciplinas de ciências, é apresentado com alunos de química do colégio mexicano, uma nova ferramenta chamada Diagrama é apresentada ABP heurística que facilita a aquisição de competência argumentativa.

Palavras-chave: Revisão Sistemática, Método $\mathrm{PBL}$, Aprendizagem de vetores. 


\section{CAPÍTULO I. INTRODUCCIÓN}

En la década pasada, el Método ABP y su influencia en el aprendizaje fue muy significativo y actualmente también son muy usados en el proceso de enseñanzaaprendizaje, así Valdés Y Ortiz (2018) señalaron que los métodos empleados son de carácter teórico y entre los principales resultados se hallan la argumentación sobre el surgimiento del ABP y la EP, definiciones propuestas por diferentes autores y sus características esenciales. Además, se enfatiza en los roles asumidos por profesores y estudiantes y se describen algunos elementos metodológicos para su posterior aplicación en el proceso de enseñanza-aprendizaje.

Moraga Negrete (2018) Una Experiencia de Aprendizaje Colaborativo, Una Adaptación del Método ABP decidió adaptar la técnica del aprendizaje basado en problemas (ABP) para aplicar la metodología en la asignatura de Derecho Tributario en la carrera de Ingeniería Comercial en la UBB sede Chillán. Los objetivos al usar este método en la asignatura eran mejorar los resultados académicos de los estudiantes, desarrollar competencias relacionadas con el trabajo en equipo y reflexión entre otras, y entregar los contenidos de manera dinámica para una mayor comprensión de los mismos.

García Retana (2012); en su artículo El lenguaje ordinario: la clave para el aprendizaje de las matemáticas basado en problemas; planteó una nueva propuesta de educación matemática para responder a las exigencias sociales y económicas actuales. Esta propuesta se fundamenta en el aprendizaje basado en problemas (ABP) como estrategia metodológica. En el caso del aprendizaje de las matemáticas, tal propuesta demanda considerar la relación que existe entre el lenguaje ordinario y el lenguaje matemático, por cuanto el primero es central en el proceso educativo. Este tipo de aprendizaje se debe conceptualizar en su doble función de herramienta, es decir, para resolver problemas, y como disciplina, dado que el lenguaje matemático permite representar los conceptos que trata, al menos de dos maneras diferentes, la semántica y la gráfico-visual, los cuales en gran medida son determinados por el lenguaje ordinario. Así, el lenguaje ordinario y su campo semántico constituyen el eje transversal para el aprendizaje de esta estrategia metodológica.

Según Llargués, Herranz, Sánchez, Calbo y Virumbrales; en el artículo Aplicación de un modelo híbrido de aprendizaje basado en problemas como estrategia de evaluación e interrelación multiasignaturas; el modelo hibrido ha sido diseñado sobre la plataforma 
de tecnología de la información Moodle que facilita su desarrollo con menos recursos. El ABP implementado funciona como una actividad integradora que trata de fomentar el desarrollo de competencias transversales y el pensamiento complejo, en un entorno de aprendizaje activo. Promueve en el estudiante la construcción de su propio conocimiento para el desarrollo de competencias como el razonamiento clínico y la toma de decisiones, al tiempo que posibilita la integración de los conocimientos procedentes de distintas asignaturas.

Adelina Mezzari en su artículo 'O uso da Aprendizagem Baseada em Problemas (ABP) como reforço ao ensino presencial utilizando o ambiente de aprendizagem Moodle"' se estableció como objetivo implementar cambios en la disciplina de Parasitología y Micología Médica de la carrera de Medicina de la Universidad Federal de Ciencias de la Salud de Porto Alegre (UFCSPA), que siempre se ha ofrecido de manera presencial. El estudio se llevó a cabo de marzo a julio de 2019 con estudiantes del primer semestre del primer año de la carrera de medicina. El cambio consistió en utilizar parte de la educación a distancia (EAD) con la aplicación del método de enseñanza ABP, en el entorno virtual Moodle, disponible en la institución. Los resultados de este estudio señalan que el uso de recursos de aprendizaje a distancia y ABP como complemento a la educación presencial de pregrado en cursos de salud puede resultar efectivo para la formación profesional.

Ante la problemática descrita, se formularon los siguientes problemas, así se tiene el problema general: ¿Cuál es la influencia del método basado en problemas (ABP) en el aprendizaje de vectores en estudiantes universitarios entre los años 2015 al $2020 ?$

Por otro lado, el objetivo conocer la influencia del método basado en problemas (ABP) en el aprendizaje de vectores en estudiantes universitarios

\section{CAPÍTULO II. METODOLOGÍA}

Se realizó una revisión sistemática de la literatura científica con bases de datos en la adaptación de la metodología PRISMA (Preferred Reporting Items for Systematic Reviews and Meta-Analyses) para la elaboración de la metodología de la revisión. Para ello, se llevaron a cabo búsquedas de estudios publicados entre 2015 al 2020, en los siguientes bases de datos electrónicos: Scopus, SciELO y ESBCO. La pregunta de 
investigación establecida para conducir el proceso metodológico fue la siguiente: ¿Cuál es la influencia del método basado en problemas (ABP) en el aprendizaje de vectores en estudiantes universitarios entre los años 2015 al $2020 ?$

La revisión de la literatura científica es una estrategia de recopilación de información que emerge ante la necesidad de conocer de manera sintética los resultados de la investigaciones para garantizar la sensibilidad del proceso de búsqueda se definieron como descriptores los siguientes términos a partir de la pregunta de investigación. En el proceso de búsqueda se definieron como descriptores los siguientes términos partir de la pregunta de investigación: "influence of the ABP method", "learning of vectors", "linear algebra course". Por la especifidad de la búsqueda de la literatura científica, se diseñó un protocolo con la combinación de los términos establecidos y los operadores booleanos: [("'influence of the ABP method" AND "'learning of vectors"') OR ("influence of the ABP method" AND "linear algebra course") OR ("learning of vectors" AND "linear algebra course")]. Del mismo modo, se definió como base de datos especializada para la búsqueda a Springerlink; como base de datos multidisciplinaria se seleccionaron Scopus, ScieLo y Ebsco que es una base de datos que ofrece textos completos, índices y publicaciones periódicas académicas que cubren diferentes áreas de las ciencias y humanidades.

Por especificidad de la búsqueda de la literatura científica se diseñó un protocolo con la combinación de los términos establecidos y los operadores booleanos para la interacción (AND) y el operador sumatorio (OR). 
En la tabla I, se expone las estrategias de búsqueda utilizadas en cada una de las bases de datos.

Tabla 1, Estrategias de búsqueda seleccionadas.

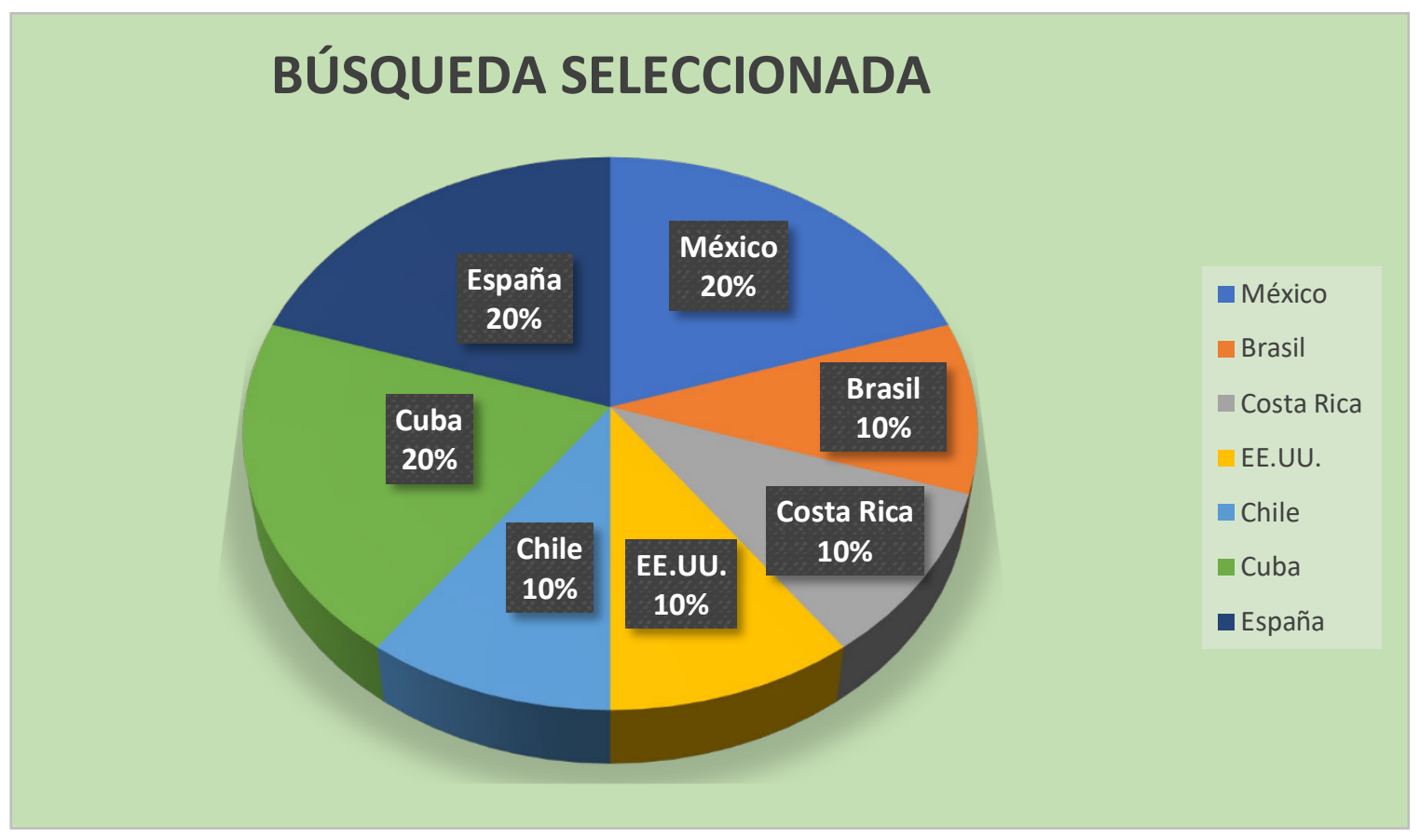

En cuanto a los criterios de inclusión estos fueron: Se logró identificar un total de 100 artículos científicos en las bases de datos mencionadas; luego se excluyeron aquellos estudios que no aportan información en relación al tema central y aquellos trabajos duplicados. , éstas se redujeron a 15 y que se refieren estrictamente a factores que influyen en el Método ABP y Aprendizaje de vectores, tal como se puede apreciar en el gráfico 1, referente al flujo de selección de unidad de análisis modelo PRISMA (Coronel et al., 2020).

Finalmente, el proceso de codificación de los datos seleccionados se realizó, trasladando éstos en una matriz respecto al nombre del artículo, referencia de la misma, año de publicación, categorías de nuestra variable de estudio (Aprendizaje de situaciones de contexto real, configuración de un plan y planteamiento de problemas aplicados a vectores), enfoque y tipo de investigación, objetivo de estudio, la población y muestra estudiada, rango de edad, género de la muestra, el instrumento empleado para medir la variable Método ABP y Aprendizaje de vectores, el resultado del estudio y el aporte alcanzado. 


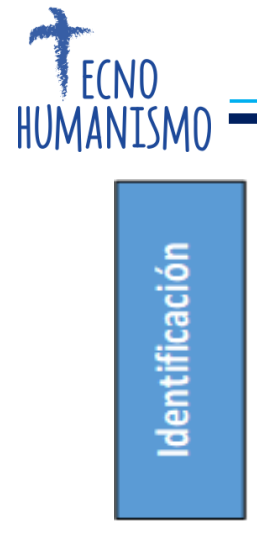

Rubén Darío Mendoza Arenas, Hugo Eladio Chumpitaz Caycho, Marisol Paola Delgado Baltazar
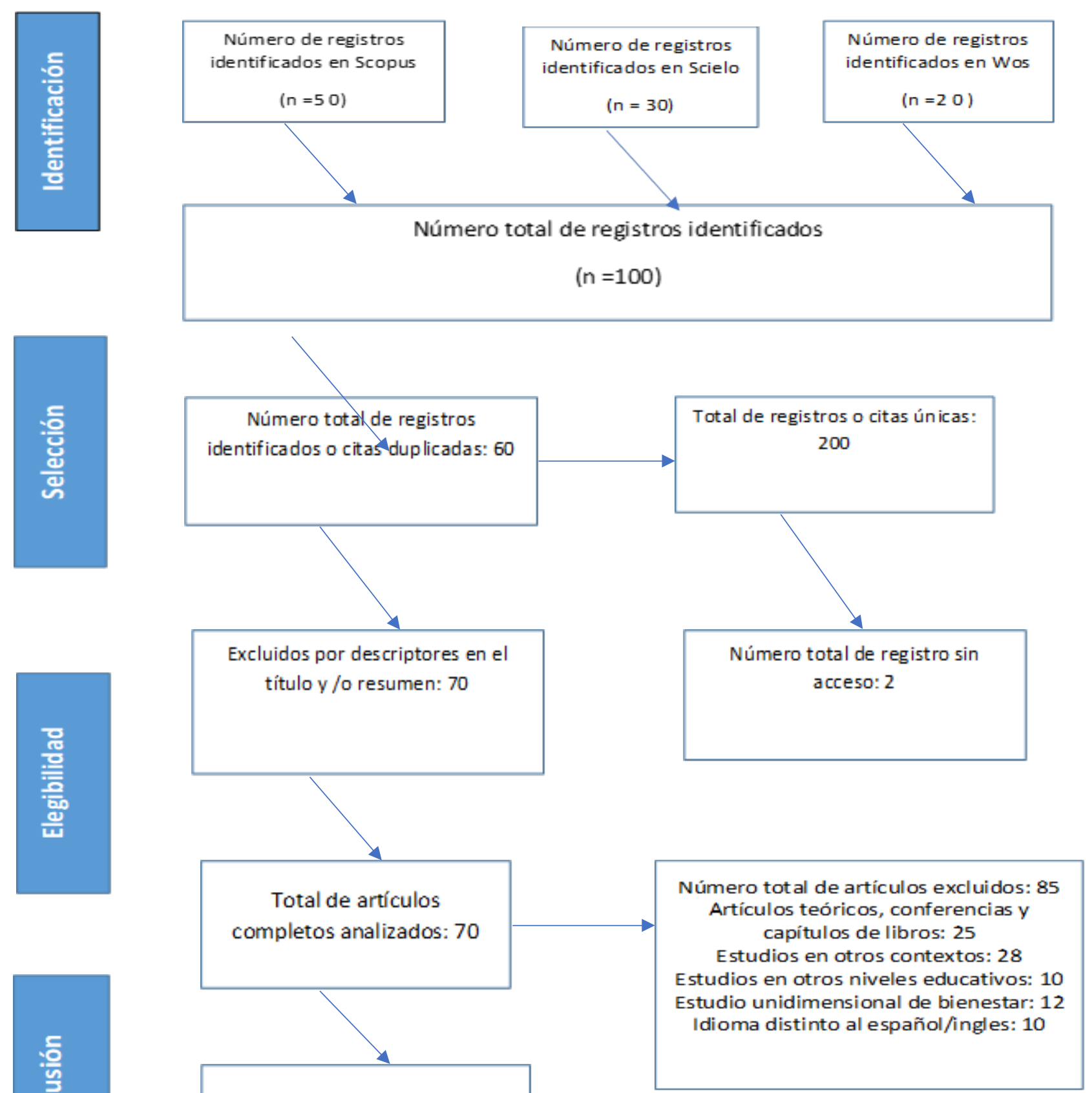

Total de artículos incluidos en

la revisión: 15

Figura 1, Diagrama de flujo para la búsqueda y selección de documentos.

\section{CAPITULO III. RESULTADOS}

El procedimiento de análisis se aplicó a los veinte estudios. Dentro de la ubicación geográfica se puede apreciar que España, Cuba y México son los países en el cual se evidencia con la mayor cantidad de publicaciones con relación al Método ABP y Aprendizaje con un total de cuatro publicaciones de cada una, luego se encuentra Brasil 
con dos, Chile con dos, EE.UU con dos y Costa Rica con dos. En cuanto al enfoque metodológico predomina el cuantitativo. (Véase Tabla 1)

Tabla 2

\begin{tabular}{|c|c|c|c|c|c|c|}
\hline AUTOR [ES] & TITULO & METODOLOGÍA & País & คคิด & LINK & BASE DE \\
\hline $\begin{array}{c}\text { Marianela } \\
\text { Moraga Negrete }\end{array}$ & $\begin{array}{c}\text { Una Experiencia } \\
\text { de Aprendizaje } \\
\text { Colaborativo. } \\
\text { Una Adaptación } \\
\text { del Método } \\
\text { ABP }\end{array}$ & $\begin{array}{c}\text { Se aplica la } \\
\text { metodología en la } \\
\text { asignatura de Derecho } \\
\text { Tributario en la carrera } \\
\text { de Ingeniería } \\
\text { Comercial. }\end{array}$ & Costa Rica & Sept.-10 & 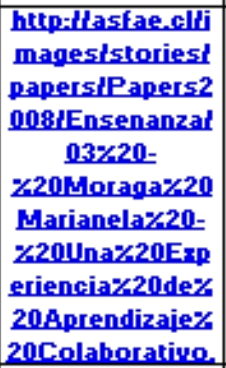 & Scielo \\
\hline $\begin{array}{c}\text { Gerardo Chávez } \\
\text { Saavedra1 } \\
\text { Beatriz Yerónica } \\
\text { González } \\
\text { Sandoval1 } \\
\text { Carlos Hidalgo } \\
\text { Yaladez1 }\end{array}$ & \begin{tabular}{|} 
Aprenulzale \\
Basado en \\
Problemas \\
(ABP) a través \\
del m-learning \\
para el abordaje \\
de casos \\
clínicos. Una \\
propuesta \\
innovadora en
\end{tabular} & $\begin{array}{l}\text { combina ambos } \\
\text { elementos en la } \\
\text { presentación. } \\
\text { discusión g solución de } \\
\text { un caso clínico real y } \\
\text { en vivo desde un } \\
\text { hospital por parte de } \\
\text { alumnos que se } \\
\text { encuentran a distancia. }\end{array}$ & Mézico & $\begin{array}{c}\text { sep.ldic. } \\
2016\end{array}$ & 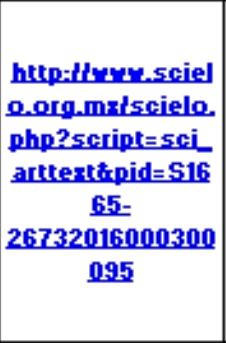 & Scielo \\
\hline $\begin{array}{c}\text { Silvia Lizett } \\
\text { Olivares } \\
\text { Olivares" y } \\
\text { Yolanda Heredia } \\
\text { Escorza"- }\end{array}$ & $\begin{array}{c}\text { Desarrollo del } \\
\text { pensamiento } \\
\text { crítico en } \\
\text { ambientes de } \\
\text { aprendizaje } \\
\text { basado en } \\
\text { problemas en } \\
\text { estudiantes de } \\
\text { educación } \\
\text { superior }\end{array}$ & $\begin{array}{c}\text { El presente estudio } \\
\text { consistió en comparar } \\
\text { los niveles de } \\
\text { pensamiento crítico } \\
\text { obtenidos con el Test } \\
\text { California de Destrezas } \\
\text { de Pensamiento } \\
\text { Crítico [CCTST-2000] } \\
\text { de Facione [2000] por } \\
\text { estudiantes de salud } \\
\text { formados con ABP } \\
\text { con alumnos de la } \\
\text { misma escuela que no }\end{array}$ & Mézico & $\begin{array}{c}\text { jul.tsep. } \\
2012\end{array}$ & $\begin{array}{l}\text { http:llwersciel } \\
\text { o.org.matscielo } \\
\text { php?pid=S1405- } \\
66662012000300 \\
004 t \text { script=sci_ } \\
\text { artert }\end{array}$ & Scielo \\
\hline
\end{tabular}




\begin{tabular}{|c|c|c|c|c|c|c|}
\hline AUTOR [ES] & TITULO & METODOLOGÍA & País & คศิก & LINK & $\begin{array}{c}\text { BASE DE } \\
\text { DATOS }\end{array}$ \\
\hline $\begin{array}{c}\text { M. Teresa } \\
\text { Alcolea-Cosín, } \\
\text { Cristina Oter- } \\
\text { Quintana, Rosa } \\
\text { M. Martínez- } \\
\text { Ortega, Tomás } \\
\text { Sebastián- } \\
\text { Viana, Azucena } \\
\text { Pedraz-Marcos }\end{array}$ & $\begin{array}{c}\text { Aprendizaje } \\
\text { basado en } \\
\text { problemas en la } \\
\text { formación de } \\
\text { estudiantes de } \\
\text { enfermería. } \\
\text { Impacto en la } \\
\text { práctica clínica }\end{array}$ & \begin{tabular}{|} 
Los resultados \\
sugieren que el uso de \\
ABP en la formación \\
académica de los \\
estudiantes de \\
enfermería no \\
modifica la puntuación \\
obtenida en su práctica \\
clínica en las \\
categorias recogidas \\
en nuestra \\
herramienta de \\
evaluación.
\end{tabular} & EEUU & $\operatorname{mar}-12$ & $\begin{array}{l}\text { htp://scielo.isci } \\
\text { ii.esiscielo.php? } \\
\text { script=sci artte } \\
\underline{x+\text { spid=S1575- }} \\
\frac{1813201200010}{\underline{0007}}\end{array}$ & Scielo \\
\hline $\begin{array}{c}\text { Eugenia } \\
\text { Saavedra R. (1) } \\
\text { Mónica Hlesca } \\
\text { P. (1) Mirtha } \\
\text { Cabezas G. (2) }\end{array}$ & $\begin{array}{l}\text { Aprendizaje } \\
\text { basado en } \\
\text { problemas } \\
\text { (ABP) como } \\
\text { estrategia para } \\
\text { adquisición de } \\
\text { competencias } \\
\text { genéricas: } \\
\text { estudiantes de } \\
\text { nutrición y } \\
\text { dietética, } \\
\text { Universidad de } \\
\text { La Frontera }\end{array}$ & $\begin{array}{l}\text { El análisis de datos } \\
\text { siguió un esquema de } \\
\text { reducción progresiva } \\
\text { de ellos, validándose } \\
\text { por triangulación de } \\
\text { investigadores. }\end{array}$ & Chile & jun. 2014 & $\begin{array}{l}\text { https://scielo.co } \\
\text { nicyt.cl/scielo.p } \\
\text { hp?pid=\$0717- } \\
7518201400020 \\
0007 \& s c r i p t=s c i \\
\text { arttext\&thg-p }\end{array}$ & Scielo \\
\hline $\begin{array}{c}\text { Hitler } \\
\text { Alexander Pinos } \\
\text { Medrano }\end{array}$ & $\begin{array}{c}\text { Uso del método } \\
\text { de aprendizaje } \\
\text { basado en } \\
\text { proyectos (Abp), } \\
\text { para la carrera } \\
\text { de arquitectura }\end{array}$ & $\begin{array}{c}\text { Usando el método de } \\
\text { aprendizaje basado en } \\
\text { problemas, se insertan } \\
\text { las TIC como opción } \\
\text { efectiva y eficaz para } \\
\text { contribuir a mejorar la } \\
\text { calidad de las } \\
\text { actividades docentes. }\end{array}$ & Cuba & $\begin{array}{c}\text { mayo.- } \\
\text { ago. } 2015\end{array}$ & $\begin{array}{l}\frac{\text { http://scielo.sld }}{\text { cu/scielo.php?sc }} \\
\frac{\text { ript=sci arttext }}{\text { ript }} \\
\frac{\text { spid=\$2218- }}{3620201500020} \\
\underline{0016}\end{array}$ & Scielo \\
\hline $\begin{array}{c}\text { Andoni } \\
\text { Arguinhano } \\
\text { Madrazol } \\
\text { Luabi Karrera } \\
\text { Juarros l } \\
\text { Maite Arandia } \\
\text { Loronol }\end{array}$ & $\begin{array}{c}\text { Funcionamient } \\
\text { o democrático y } \\
\text { ABP: factores } \\
\text { determinantes } \\
\text { para la } \\
\text { inclusión y el } \\
\text { rendimiento } \\
\text { del alumnado }\end{array}$ & \begin{tabular}{|} 
Este trabajo analiza \\
cómo el \\
funcionamiento \\
democrático y la \\
metodología basada en \\
proyectos (ABP) \\
influyen en el \\
rendimiento del \\
alumnado.
\end{tabular} & EEUU & $\begin{array}{c}\text { abr./jun. } \\
2018\end{array}$ & 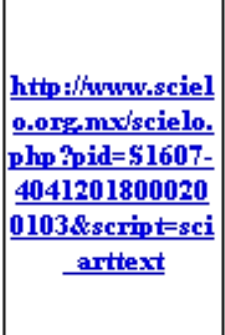 & Scielo \\
\hline
\end{tabular}




\begin{tabular}{|c|c|c|c|c|c|c|}
\hline AUTOR [ES] & TITULO & METODOLOGÍA & País & คศึก & LINK & $\begin{array}{c}\text { BASE DE } \\
\text { DATOS }\end{array}$ \\
\hline $\begin{array}{c}\text { Rocío Carolina } \\
\text { García Riveraa } \\
\text { Adrián Martínez } \\
\text { Gonzáleza * }\end{array}$ & $\begin{array}{c}\text { Calidad de los } \\
\text { problemas de } \\
\text { ABP. Evidencia } \\
\text { de validez de } \\
\text { un instrumento }\end{array}$ & $\begin{array}{c}\text { El instrumento fue } \\
\text { desarrollado sobre la } \\
\text { base de la revisión de } \\
\text { la literatura. Se } \\
\text { obtuvieron evidencias } \\
\text { de validex de acuerdo } \\
\text { con los Standards. }\end{array}$ & Mexico & $\begin{array}{c}\text { ene./mar. } \\
2019\end{array}$ & 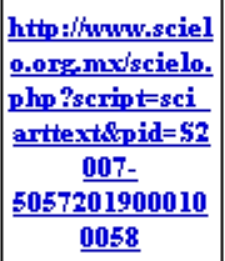 & Scielo \\
\hline $\begin{array}{c}\text { Emilio Zhuma } \\
\text { Meral }\end{array}$ & $\begin{array}{c}\text { Estrategias de } \\
\text { trabajo } \\
\text { colaborativo } \\
\text { ABP - TPA }\end{array}$ & $\begin{array}{c}\text { El presente estudio } \\
\text { presenta una } \\
\text { estrategia } \\
\text { metodológica } \\
\text { implementada en la } \\
\text { carrera Ingeniería en } \\
\text { Telemática. }\end{array}$ & Cuba & $\begin{array}{c}\text { abr.-jun. } \\
2019\end{array}$ & $\begin{array}{l}\frac{\text { http://scielo.sld }}{\text { cu/scielo.php?sc }} \\
\frac{\text { ript=sci arttext }}{\underline{\text { spid }=\$ 2218-}} \\
\frac{\underline{3620201900020}}{\underline{0153}}\end{array}$ & Scielo \\
\hline $\begin{array}{l}\text { Suellen Fabiane } \\
\text { CamposI; } \\
\text { Angela } \\
\text { Conceiçăo } \\
\text { Pereira } \\
\text { ChagasI; Aline } \\
\text { Bárbara Pereira } \\
\text { CostaI; Rosilene } \\
\text { Estevăo de Melo } \\
\text { Françall; Ann } \\
\text { Kristine JansenI }\end{array}$ & \begin{tabular}{|c|} 
Fatores \\
associados ao \\
desenvolviment \\
o de úlceras de \\
pressăo: o \\
impacto da \\
nutriçắo
\end{tabular} & $\begin{array}{c}\text { Verificou-se a } \\
\text { predominância de } \\
\text { individuos do sexo } \\
\text { feminino, com média } \\
\text { de idade de } 66,6, \\
\text { DP=18,0 anos. A } \\
\text { incidência das injúrias } \\
\text { foi de } 28,0 \% \text {, } \\
\text { resultando em uma } \\
\text { média de } 1,7 \text { feridas } \\
\text { por paciente, mais } \\
\text { frequentes na regiăo } \\
\text { sacral ( } 57,1 \% \text { ) em } \\
\text { calcanhares (35,7\%) }\end{array}$ & Brasil & $\begin{array}{l}\text { Sept/Oct } \\
.2010\end{array}$ & \begin{tabular}{|}
$\frac{\text { https://www.scie }}{\text { lo.briscielo.php }}$ \\
$\frac{\text { 2pid=\$1415- }}{\text { 5273201000050 }}$ \\
$\frac{0002 \& \text { scriptsci }}{\text { arttext }}$
\end{tabular} & Scielo \\
\hline $\begin{array}{c}\text { Gregori Giralt, } \\
\text { Evaa; Menéndex } \\
\text { Varela, José } \\
\text { Luisb }\end{array}$ & $\begin{array}{c}\text { La evaluación } \\
\text { en el } \\
\text { Aprendizaje } \\
\text { Basado en } \\
\text { Problemas } \\
\text { (ABP) } \\
\text { Resultados de } \\
\text { una } \\
\text { experiencia } \\
\text { didáctica en los } \\
\text { estudios } \\
\text { universitarios } \\
\text { de Bellas Artes }\end{array}$ & $\begin{array}{c}\text { Este trabajo analiza } \\
\text { las calificaciones } \\
\text { obtenidas por } 247 \\
\text { estudiantes de los } \\
\text { primeros semestres de } \\
\text { la titulación de Bellas } \\
\text { Artes que elaboraron } \\
\text { una carpeta de } \\
\text { aprendizaje asociada a } \\
\text { un entorno de } \mathrm{ABP} \text {. }\end{array}$ & Espan̆a & $\max -15$ & $\begin{array}{l}\frac{\text { htps://scielo.co }}{\text { nicytcl/scielo.p }} \\
\underline{\text { hp?pid=S0718- }} \\
\underline{0705201500020} \\
0006 \& s c r i p t=s c i \\
\text { arttext\&tlng-p }\end{array}$ & Scielo \\
\hline
\end{tabular}




\begin{tabular}{|c|c|c|c|c|c|c|}
\hline AUTOR [ES] & TITULO & METODOLOGía & País & คศึั & LINK & $\begin{array}{c}\text { BASE DE } \\
\text { DATOS }\end{array}$ \\
\hline $\begin{array}{l}\text { Dayana Travieso } \\
\text { Valdés, Tania } \\
\text { Ortiz Cárdenas }\end{array}$ & \begin{tabular}{|c|} 
Aprendizaje \\
basado en \\
problemas y \\
enseñanza por \\
proyectos: \\
alternativas \\
diferentes para \\
ensen̆ar
\end{tabular} & \begin{tabular}{|} 
En este sentido, el \\
objetivo fundamental \\
es caracterizar el \\
aprendizaje basado en \\
problemas (ABP) y la \\
enseñanza por \\
proyectos (EP) como \\
tendencias de \\
formación \\
universitaria.
\end{tabular} & Cuba & $\begin{array}{c}\text { ene.-abr. } \\
2018\end{array}$ & 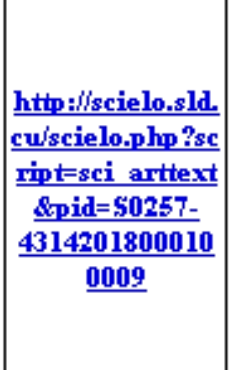 & Scielo \\
\hline $\begin{array}{c}\text { Yosajandi Pérex } \\
\text { CampilloI; José } \\
\text { Antonio } \\
\text { Chamizo } \\
\text { GuerreroII }\end{array}$ & \begin{tabular}{|c|} 
El ABP y el \\
diagrama \\
heuristico como \\
herramientas \\
para \\
desarrollar la \\
argumentación \\
escolar en las \\
asignaturas de \\
ciencias
\end{tabular} & \begin{tabular}{|c|} 
En la presente \\
investigación, \\
enmarcada en el \\
Aprendizaje Basado en \\
Problemas, (ABP) \\
sobre el tema de \\
minerales con \\
alumnos de química \\
del bachillerato \\
mexicano.
\end{tabular} & Brasil & jul-13 & 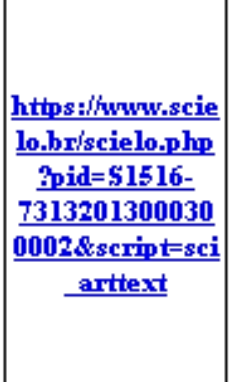 & Scielo \\
\hline $\begin{array}{l}\text { Esteve Llargués, } \\
\text { Xavier Herranz, } \\
\text { Lluis Sánchex, } \\
\text { Esther Calbo, } \\
\text { Montserrat } \\
\text { Virumbrales }\end{array}$ & \begin{tabular}{|c|} 
Aplicación de \\
un modelo \\
hibrido de \\
aprendizaje \\
basado en \\
problemas \\
como estrategia \\
de evaluación e \\
interrelación \\
'multiasignatur \\
as'
\end{tabular} & $\begin{array}{l}\text { El modelo hibrido ha } \\
\text { sido diseñado sobre la } \\
\text { plataforma de } \\
\text { tecnología de la } \\
\text { información Moodle } \\
\text { que facilita su } \\
\text { desarrollo con menos } \\
\text { recursos. }\end{array}$ & Espan̆a & $\begin{array}{c}\text { mar./abr. } \\
2015\end{array}$ & $\begin{array}{c}\text { http://scielo.isci } \\
\text { ii.es/scielo.php? } \\
\text { pid=\$2014 } \\
\underline{9832201500020} \\
0009 \& \text { script=sci } \\
\text { arttext\&tlng=e } \\
\underline{n}\end{array}$ & Scielo \\
\hline $\begin{array}{l}\text { Daniela da Silva } \\
\text { RochaI; Joel } \\
\text { Alves } \\
\text { LamounierII; } \\
\text { Flávio Diniz } \\
\text { CapanemaII; } \\
\text { Sylvia do Carmo } \\
\text { C. } \\
\text { Franceschinirv; } \\
\text { Rocksane de } \\
\text { Carvalho } \\
\text { NortonV }\end{array}$ & \begin{tabular}{|c|} 
Estado \\
nutricional e \\
prevalência de \\
anemia em \\
crianças que \\
freqüentam \\
creches em \\
Belo Horizonte, \\
Minas Gerais
\end{tabular} & \begin{tabular}{|c|} 
Estudo transversal em \\
25 creches da regional \\
leste de Belo \\
Horizonte, Minas \\
Gerais. Realizaram-se \\
avaliaçăo \\
antropométrica e \\
dosagem de \\
hemoglobina por \\
hemoglobinômetro \\
portátil.
\end{tabular} & Brasil & $\begin{array}{l}\text { Mar. } \\
2008\end{array}$ & $\mid \begin{array}{l}\text { https://hww.scie } \\
\frac{\text { lo.briscielo.php }}{\text { 2pid=S0103- }} \\
\underline{0582200800010} \\
\frac{0002 \& \text { script=sci }}{\text { arttext }} \\
\end{array}$ & Scielo \\
\hline
\end{tabular}




\begin{tabular}{|c|c|c|c|c|c|c|}
\hline AUTOR [ES] & TITULO & METODOLOGía & País & Aศกั & LINK & $\begin{array}{c}\text { BASE DE } \\
\text { DATOS }\end{array}$ \\
\hline Adelina Mexzari & \begin{tabular}{|c|} 
O uso da \\
Aprendizagem \\
Baseada em \\
Problemas \\
(ABP) como \\
reforço ao \\
ensino \\
presencial \\
utilizando o \\
ambiente de \\
aprendizagem \\
Moodle
\end{tabular} & \begin{tabular}{|} 
Este estudo teve por \\
objetivo implementar \\
mudanças na \\
disciplina de \\
Parasitologia e \\
Micologia Médica do \\
curso de Medicina da \\
Universidade Federal \\
de Ciências da Saúde \\
de Porto Alegre \\
(UFCSPA), que sempre \\
foi oferecida na \\
modalidade presencial.
\end{tabular} & Brasil & $\begin{array}{c}\text { Jan./Mar. } \\
2011\end{array}$ & $\begin{array}{c}\text { https://www.scie } \\
\text { lo.briscielo.php } \\
\text { 3pid=\$0100- } \\
\underline{502201100010} \\
\underline{0016 \& \text { script=sci }} \\
\text { arttext\&thgep } \\
\underline{t}\end{array}$ & Scielo \\
\hline $\begin{array}{c}\text { García Retana, } \\
\text { José Ángel }\end{array}$ & $\begin{array}{c}\text { El lenguaje } \\
\text { ordinario: la } \\
\text { clave para el } \\
\text { aprendizaje de } \\
\text { las } \\
\text { matemáticas } \\
\text { basado en } \\
\text { problemas } \\
\end{array}$ & \begin{tabular}{|c} 
Esta propuesta se \\
fundamenta en el \\
aprendizaje basado en \\
problemas (ABP) como \\
estrategia \\
metodológica. En el \\
caso del aprendizaje \\
de las matemáticas, tal \\
propuesta demanda \\
considerar la relación \\
que existe entre el \\
lenguaje ordinario y el \\
lenguaje matemático
\end{tabular} & Costa Rica & ago-13 & $\begin{array}{l}\text { http://repositori } \\
\text { o.ucr.ac.cr/hand } \\
\text { le/10669/17144 }\end{array}$ & Scielo \\
\hline $\begin{array}{c}\text { José Osvaldo } \\
\text { Enriquex } \\
\text { Clavero }\end{array}$ & \begin{tabular}{|c|} 
¿Ensen̆anza \\
problémica es \\
igual a \\
aprendizaje \\
basado en \\
problemas? Un \\
ejemplo en \\
Morfofisiología
\end{tabular} & $\begin{array}{c}\text { En un curso pre-evento } \\
\text { impartido } \\
\text { recientemente con el } \\
\text { propósito de estimular } \\
\text { el uso de la Enseñanza } \\
\text { Problémica (EP) como } \\
\text { una alternativa } \\
\text { metodológica viable en } \\
\text { la educación médica } \\
\text { superior. }\end{array}$ & Cuba & feb-14 & $\begin{array}{l}\frac{\text { http://scielo.sld }}{\text { cu/scielo.php?sc }} \\
\frac{\text { ript=sci arttext }}{\text { sid }} \\
\frac{\text { spid=\$2077- }}{2874201400040} \\
\underline{0014}\end{array}$ & Scielo \\
\hline $\begin{array}{c}\text { Antonio L. } \\
\text { García R.1, José } \\
\text { A. Jiménez L.1, } \\
\text { Efrén } \\
\text { Rodriguez? }\end{array}$ & $\begin{array}{l}\text { Bases teóricas } \\
\text { del modelo de } \\
\text { principios } \\
\text { científico- } \\
\text { didácticos para } \\
\text { la enseñanza } \\
\text { de la geografia } \\
\text { y de la historia }\end{array}$ & $\begin{array}{c}\text { El modelo } \\
\text { metodológico basado } \\
\text { en la implementación } \\
\text { de Principios } \\
\text { Científico-Didácticos } \\
\text { en la enseñanza de la } \\
\text { Geografia y de la } \\
\text { Historia. }\end{array}$ & Espan̆a & jun. 2009 & 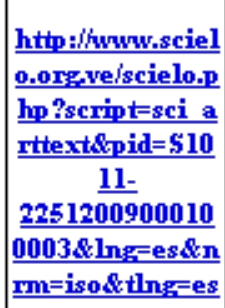 & Scielo \\
\hline
\end{tabular}


Tabla 3

La variable Método ABP

\begin{tabular}{|c|c|c|}
\hline AUTOR (ES) & TÍTULO & NIVEL \\
\hline Íñigo Noriega Echevarría & $\begin{array}{l}\text { Nuevas metodologías, la } \\
\text { innovación como estrategia: el } \\
\text { punto de vista de los estudiantes }\end{array}$ & Bajo \\
\hline $\begin{array}{c}\text { Yuriet Verde Trujillo } \\
\text { Tomás Cañas Louzau }\end{array}$ & $\begin{array}{l}\text { El aprendizaje basado en } \\
\text { problemas en la apreciación de } \\
\text { las artes plásticas }\end{array}$ & Medio \\
\hline $\begin{array}{c}\text { Alexander López Padrón } \\
\text { Mamilet Irene Gutiérrez Gaitén } \\
\text { Amelia Teresina Henriques } \\
\text { Gloria del Barrio Alonso }\end{array}$ & $\begin{array}{l}\text { Análisis de flavonoides en una } \\
\text { fracción butanólica obtenida de } \\
\text { Phyllanthus orbicularis HBK }\end{array}$ & Alto \\
\hline
\end{tabular}

Tabla 4

La variable Aprendizaje de Matemáticas

\begin{tabular}{|l|l|l|}
\hline \multicolumn{1}{|c|}{ AUTOR (ES) } & \multicolumn{1}{c|}{ TÍTULO } & NIVEL \\
\hline Cadena Zambrano, Verónica E & $\begin{array}{l}\text { Aprendizaje basado en } \\
\text { problemas aplicado en } \\
\text { Matemática }\end{array}$ & Medio \\
\hline $\begin{array}{l}\text { Marcos Campos Nava, Agustín } \\
\text { Alfredo Torres Rodríguez }\end{array}$ & $\begin{array}{l}\text { Empleo de un videojuego como } \\
\text { recurso didáctico en la clase de } \\
\text { matemática: el caso del puzzle } \\
\text { hands of time }\end{array}$ & Bajo \\
\hline $\begin{array}{l}\text { Carina Lorena Fernández } \\
\text { María Inés Aguadoa }\end{array}$ & $\begin{array}{l}\text { Aprendizaje basado en } \\
\text { problemas como complemento } \\
\text { de la enseñanza tradicional en } \\
\text { Fisicoquímica }\end{array}$ & Alto \\
\hline
\end{tabular}

\section{CAPÍTULO IV. DISCUSIÒN Y CONCLUSIONES}

Esta revisión sistemática ha recogido veinte estudios empíricos sobre las variables, Método ABP y Aprendizaje de Vectores, trae como consecuencia un ambiente de generación de aprendizajes. (Badillo, M.; Bonilla, M. y Paredes, L. 2018) El ABP consiste 
en el planteamiento de una situación problema, donde su construcción, análisis y/o solución constituyen el foco central de la experiencia, y donde la enseñanza consiste en promover deliberadamente el desarrollo del proceso de indagación y resolución del problema en cuestión. Mientras en el modelo tradicional de enseñanza, el profesor expone primero la información y posteriormente busca su aplicación en la resolución del problema, en el método ABP se presenta el problema, se identifica la necesidad del aprendizaje, se busca la información necesaria para la resolución del problema y finalmente se resuelve. En este método tiene vital importancia la adquisición de conocimientos como el desarrollo de habilidades y actitudes.

El Centro para el Aprendizaje Basado en problemas de la Academia de Matemáticas y Ciencias de Illinois (Center for Problem Based Learning, Illinois Mathematics and Science Academy) desarrolló un modelo y aplicación del método ABP en la educación básica y media, este modelo plantea dos procesos básicos interrelacionados $\mathrm{y}$ complementarios en el ABP: El diseño curricular y el entrenamiento cognitivo.

Según Paredes-Curin en el 2016 ; el aprendizaje basado en problemas se basa en la paradigma constructivista que centra su proceder en la acción del alumnado, donde este el que realiza su propio aprendizaje. La temática a tratar en este estudio es la aplicación de este método en educación media, en un establecimiento con un alto índice de vulnerabilidad (90\%) de la comuna de Cañete, octava región de Chile. El ABP, según el estado del arte ha sido desarrollado mayormente en el ambiente de la educación formal superior en carreras como medicina y en estudios de recursividad.

Cecilia Jofre y Fernando Contreras en el 2016, en su artículo Implementación de la Metodología ABP (Aprendizaje Basado en Problemas) en Estudiantes de Primer año de la Carrera de Educación Diferencial, el desarrollo de destrezas y competencias individuales en contextos formales se ve beneficiado por la interacción con otros. No basta sólo con presentar determinadas actividades de aprendizaje, es necesario organizar el contexto en el cual el aprendizaje ocurre con el fin de optimizar el proceso y potenciar las destrezas de los alumnos En este sentido, en la búsqueda de un método didáctico generador de conocimientos significativos y de habilidades, se presenta el Método de Aprendizaje Basado en Problemas (ABP) como una alternativa efectiva para ser implementada en carreras de Pedagogía. 
Un antecedente del ABP en Medicina es el que presenta y expone el ABP es una técnica didáctica centrada en el alumno. En ella el docente plantea un problema clínico inicial, complejo y retador para que sea resuelto basándose en el trabajo colaborativo dentro de grupos de trabajo pequeños, con el objeto de desencadenar el aprendizaje autodirigido). Se empezó a utilizar por primera vez en la escuela de medicina de la Universidad de Case Western Reserve en los Estados Unidos, y en la Universidad de Mc Master en Canadá en la década de los 60 con el objetivo de mejorar la calidad de la educación. El proceso del aprendizaje convencional se invierte al trabajar con el ABP: primero se presenta el problema (casos clínicos), se identifican las necesidades de aprendizaje, se busca la información necesaria y finalmente se regresa al problema para resolverlo (Chávez Saavedra y González Sandoval; 2016)

Por lo tanto, se pone en evidencia la interrelación de ambos aspectos pueden servir de línea de investigación para futuros trabajos. En relación con los objetivos de investigación, según los estudios empíricos realizados, en base al Método ABP y el aprendizaje se determinó de acuerdo al procedimiento de análisis que se aplicó a los veinte estudios. Dentro de la ubicación geográfica se puede apreciar que España, Cuba y México son los países en el cual se evidencia con la mayor cantidad de publicaciones con relación al Método ABP y Aprendizaje con un total de cuatro publicaciones de cada una, luego se encuentra Brasil con dos, Chile con dos, EE.UU con dos y Costa Rica con dos, en el cual predomina el método cuantitativo. Por todo ello se determinó que en su mayoría, son cuatro países donde se evidencia mayor cantidad de publicaciones con relación al Método ABP y aprendizaje. En tal sentido es necesario que los docentes utilicen dichas publicaciones con el fin de lograr aplicar mejores estrategias en base al Método ABP y aprendizaje a fin de garantizar un ambiente de armonía y trabajo colectivo, asimismo puedan orientar la resolución de problemas dentro de su contexto social.

\section{Referencias}

Barrows (1986). A Taxonomy of problem-based learning methods, en Medical Education, 20/6, 481-486.

Bernal (2006). Metodología de la Investigación. Tercera Edición. PEARSON, Universidad La Sabana, Colombia. 
Castillo, S. y Cabrerizo, J. (2010) Evaluación Educativa de aprendizajes. MadridEspaña. PEARSON EDUCACIÓN, S.A.

Corominas (1987). Breve diccionario etimológico de la lengua castellana. Madrid: Gredos. Corripio (1984). Diccionario etimológico general de la lengua castellana. Barcelona:Bruguera.

De La Rosa (2016) en su tesis titulada Aprendizaje basado en problemas como estrategia metodológica para mejorar el rendimiento académico en la asignatura de complemento matemático en los estudiantes de la Escuela Profesional de Ingeniería Industrial de la Universidad Privada Antenor Orrego; para obtener el grado de Maestro en Educación; sustentada en la Escuela de Postgrado de la Universidad Privada Antenor ORREGO, Trujillo-Perú.

De Miguel (coord.). Metodologías de enseñanza para el desarrollo de competencias. Orientaciones para el profesorado universitario ante el Espacio Europeo de Educación Superior. Madrid: Alianza.

Exley, K. y Dennis, R. (2007). Enseñanza en pequeños grupos en Educación Superior. Madrid: Narcea.

Fernández, F. y Duarte, J. (2013) en su tesis titulada El aprendizaje basado en problemas como estrategia para el desarrollo de competencias específicas en estudiantes de ingeniería. Colombia.

González, M. y I. Ramírez (2011). La formación de competencias profesionales: un reto en los proyectos curriculares universitarios. Odiseo, Revista Electrónica de Pedagogía.

Hernández, R., Fernández. y Baptista, M. (2003). Metodología de la Investigación. Sexta Edición. Mc Graw Hill. México, Bogotá, Buenos Aires, Caracas, Guatemala.

Hernández, R., Fernández. y Baptista, M. (2017). Metodología de la Investigación. Sexta Edición. Mc Graw Hill. México, Bogotá, Buenos Aires, Caracas, Guatemala.

Martínez, A.; Cegarra, J. y; Rubio, J. (2012) en su tesis titulada Aprendizaje Basado en competencias: Una propuesta para la autoevaluación del docente Profesorado. Revista 
de Currículum y Formación de Profesorado, vol. 16, núm. 2, mayo-agosto, 2012, pp. 325338. Universidad de Granada, España.

Mendoza (2015). Tesis sobre aprendizaje basado en problemas (ABP).

Mejía (2005). Metodología de la Investigación Científica. Primera Educación. Centro de Producción Editorial e Imprentade la Universidad Nacional Mayor de San Marcos. Lima-Perú.

Morales, P. y Landa, V. (2004). Aprendizaje Basado el Problemas. Theoría, 13, 145-157. Ministerio de Educación (2009), Diseño Curricular Nacional de Educación Básica Regular, Lima-Perú.

Ministerio de Educación (2016). Currículo Nacional de la Educación Básica. Perú. http://www.minedu.gob.pe/curriculo/pdf/curriculo-nacional-2016-2.pdf

Morales, P. y Landa, V. (2004). Aprendizaje basado en problemas, en Theoria, Vol.13. Págs.145-157.

Normas y orientaciones para la elaboración de programas y guías de aprendizaje. Documento interno. Universidad de Deusto, 2006.

Perrenoud (2004) La de los campos sociales: competencias del autor autónomo, en D.S. Rychen y L. H. Sasganik (eds.) Definir y seleccionar las competencias fundamentales para la vida. México. FC, 216-261.

Poblete (2007). Desarrollo de competencias y créditos trasferibles: Experiencia multidisciplinar en el contexto universitario. Universidad Politécnica de Madrid-España. Editorial: Mensajero.

Prieto (2006). Aprendizaje activo en el aula universitaria: el caso del aprendizaje basado en problemas, en Miscelánea Comillas. Revista de Ciencias Humanas y Sociales Vol.64. Núm.124. Págs. 173-196.

Ramírez, M., Pérez, E. y Tapia, F. (2015). Secuencias Didácticas para el Desarrollo de Competencias en educación media superior y superior. México: Trillas. 
Retrepo (2005). Aprendizaje basado en problemas (ABP): Una innovación didáctica para la enseñanza universitaria. Educación y Educadores. Universidad de La Sabana, Cundinamarca, Colombia.

Román (2005) Sociedad del conocimiento y refundación de la escuela desde el aula. Madrid. EOS.

Tobón (2008). La formación basada en competencias en la Educación Superior: El enfoque complejo. México-Guadalajara. Bogotá: Instituto Cife w

Tobón (2006). Las competencias en la educación superior. Políticas de calidad.

\section{Bogotá: ECOE.}

Tobón (2004). Formación basada en competencias: Pensamiento complejo, diseño curricular y didáctica. Bogotá: ECOE.

Universidad Politécnica (2008). Aprendizaje Basado en Problemas. Madrid. España.

Vizcarro, C. y Juárez, E. (2010). Capítulo 1: ¿Qué es y cómo funciona el aprendizaje Basado en Problemas? En Prieto, La Metodología del Aprendizaje Basado en Problemas. (pp.9-32).Murcia: Universidad de Murcia. 